\title{
Lumps and Bumps of the Abdominal Wall and Lumbar Region-Part 1: Hernias, What the Radiologist Should Know
}

\author{
Sangoh Lee ${ }^{1}$ Sarah R. Hudson ${ }^{1}$ Catalin V. Ivan ${ }^{1} \quad$ Tahir Hussain $^{1} \quad$ Ratan Verma $^{1} \quad$ Arumugam Rajesh ${ }^{1}$ \\ James A. Stephenson ${ }^{1}$ \\ ${ }^{1}$ Gastrointestinal Imaging Group, Department of Radiology, \\ University Hospitals of Leicester NHS Trust, Leicester General \\ Hospital, Leicester, United Kingdom

\begin{abstract}
Address for correspondence James A. Stephenson, MD, FRCR, Gastrointestinal Imaging Group, Department of Radiology, University Hospitals of Leicester NHS Trust, Leicester General Hospital, Leicester LE5 4PW, United Kingdom (e-mail: james.stephenson@uhl-tr.nhs.uk).
\end{abstract}

J Gastrointestinal Abdominal Radiol ISGAR 2018;1:12-18

\section{Introduction}

Abdominal hernias occur when part of or an organ of a body cavity protrudes through a defect in the wall of that cavity. ${ }^{1}$ It is a common condition with lifetime risk of developing a groin hernia estimated at $27 \%$ for men and $3 \%$ for women. ${ }^{2}$

External hernias that protrude through the abdominal wall are classified by their location: groin (inguinal and femoral), ventral (umbilical and paraumbilical), lumbar, and incisional hernias. These also include rare hernias such as interparietal (Richter, Littre Maydl, and Amyand) and pelvic hernias (sciatic, obturator, and perineal) ${ }^{3}$ ( - Fig. 1). Symptoms of hernias are wide ranging and are largely dependent on the location and the content of the hernia. In up to one-third of patients, a hernia remains asymptomatic. ${ }^{4}$ Others may experience several symptoms such as pain, vomiting, distension, or a dragging sensation that can be exacerbated by coughing, micturition, defecation, exercise, or sexual intercourse. ${ }^{1}$ Clinical signs include a tender abdominal mass, dehydration, and eventual peritonism. ${ }^{5}$

Entrapment of bowel into the hernia sac can cause bowel obstruction; if these hernias remain irreducible or strangulated, complications can occur as the blood supply to the affected bowel becomes compromised.

Imaging studies were previously only used when the typical expected signs and symptoms of hernias were absent; however, many surgeons now prefer to have confirmatory imaging prior to invasive corrective surgery. Moreover, imaging can be particularly useful in assessing large abdominal wall defects with involvement of different abdominal anatomical boundaries/walls (such as large incisional hernias in patients with multiple abdominal surgeries) and involving loss of domain. Imaging (particularly cross-sectional imaging) can add significant value to the process of preoperative planning in terms of helping the surgeon decide what type of surgery can be performed (laparotomy vs. laparoscopy-a key factor in the future outcome of the repair and patient morbidity), in choosing the best surgical approach but also in ensuring the correct size and type of mesh and surgical equipment are available. Imaging (especially the radiology report) can offer the surgeons a descriptive tridimensional representation of the abdominal wall defect and of the hernia sac contents helping with decision making.

We present our experience of hernia imaging of the abdominal wall and lumbar region presenting their variable imaging characteristics and related complications.

\section{Imaging of Abdominal Wall Hernias}

The primary diagnosis of a hernia can usually be made by physical examination, with imaging studies only necessitated when typical physical signs and symptoms are absent.
License terms

accepted after revision

October 5, 2018 


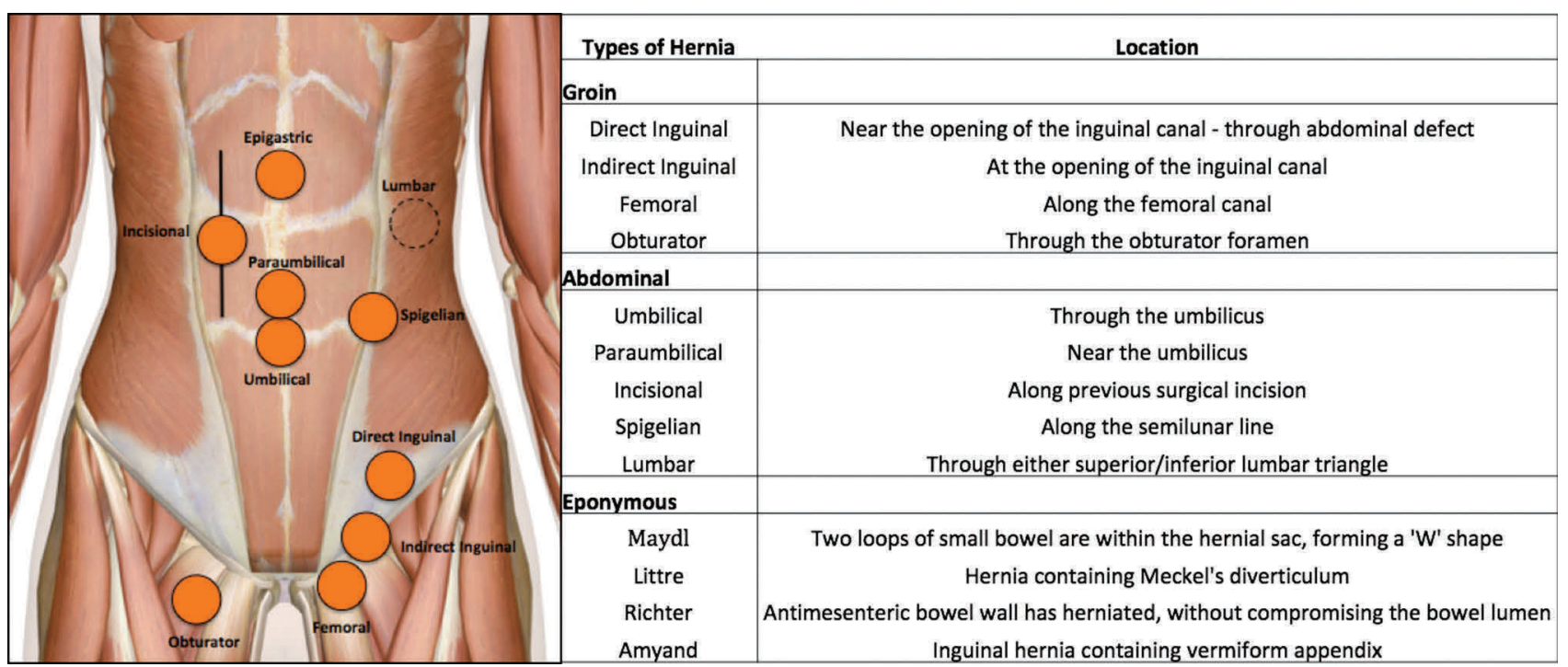

Fig. 1 Hernia types.

An ultrasound scan (USS) is the first-line investigation ${ }^{6}$ with which a good superficial anatomical resolution and dynamic assessment can be made. The US assessment of the hernia orifices should be done using linear high-frequency probes to begin with (range: $6-15 \mathrm{MHz}$ ). This helps define the anatomical detail of the abdominal wall and of the hernia sac and its contents. If the hernia sac contains bowel, the appearance of it should be further assessed and described (e.g., bowel wall thickening and loss of the definition of the bowel wall layers indicating possible ischemic changes or strangulation). The presence or absence of free fluid in the hernia sac should also be indicated in the report. Moreover, assessment of the vascularity of the herniated bowel with Doppler should be strongly considered; the vascularity, along with the morphologic changes of the herniated abdominal content can help diagnose a strangulated hernia (acute finding). A general review of the abdomen (e.g., for free fluid) can be done with the curvilinear probe $(1-5 \mathrm{MHz})$. These probes can also be used for larger hernias. Key to this is 3D spatial understanding of the anatomy in the region of interest, especially for groin hernias. The area of interest is assessed with the patient supine to identify a defect and/or a protrusion. Maneuvers to increase intra-abdominal pressure can be used to help accentuate protrusions as well as scanning patients in a standing position.

Multidetector computed tomography (CT) and magnetic resonance imaging (MRI) provide excellent anatomical detail and have the highest specificity and sensitivity. ${ }^{7}$

In our institution, the assessment of hernias on a CT scan involves a portal phase post intravenous (IV) contrast scan with thin, 1-mm slices covering the abdomen and pelvis and the area of interest (if not already covered by the protocol, e.g., in the case of a femoral hernia). Axial, coronal, and sagittal reformats are transferred to the picture archiving and communication system (PACS). Depending on the clinical scenario, if the patient has acute kidney injury with an estimated glomerular filtration rate (eGFR) $<30$, after discussion with the clinical team contrast may be withheld. IV contrast is particularly helpful in assessing the enhancement of the herniated bowel wall, especially in incarcerated hernias or closed-loop obstructions.

In younger patients, a low-dose CT protocol with IV contrast may be used. This uses low milliamperesecond ( $\mathrm{mAs}$ ). Consequently, the patient radiation dose is decreased at the detriment of image quality. However, a balanced approach may decrease the dose to up to onethird of that of a normal scan and provide adequate image quality, allowing the correct interpretation of the findings.

CT cannot provide functional assessment of hernias that USS and, more recently, dynamic MRI are able to provide. ${ }^{8}$ CT predominantly aids the assessment of the hernia content and is commonly used acutely to assess for complications. In our institution, we reserve MRI (fast-spin echo and fast gradient echo sequences) for cases with a high clinical suspicion of a hernia, but it has not been identified on USS. Sequences are acquired in axial and sagittal planes with and without straining maneuvers. In the case of the later (dynamic MRI), the scan is performed in the plain that best anatomically demonstrates the hernia by using a steadystate sequence such as FIESTA (fast imaging employing steady-state acquisition) that is capable of acquiring an image per second, which can then be reviewed in a cineloop. The standard static MRI protocol includes T1 and T2 fat-saturated axial and sagittal sequences, covering the region of interest. To focus on a region of interest, a skin marker is often used to help identify the area of clinical suspicion.

MRI and CT are also used to assess complex incisional hernias prior to abdominal wall reconstruction where domain loss can be assessed.

\section{Groin Hernias}

Careful distinction is required of hernias in the groin specifically direct inguinal, indirect inguinal, and femoral hernias due to differences in their predisposition and management. 
A direct inguinal hernia occurs as a result of weakness in the transversalis fascia in Hesselbach's triangle. This is common in the elderly and those with increased abdominal pressure, for example, patients with chronic obstructive pulmonary disease, chronic constipation, and bladder outflow obstruction. These directly protrude through the inguinal wall, medial to the epigastric vessels (-Fig. 2). An indirect inguinal hernia is five times more common than a direct inguinal hernia and seven times more common in males. This type of hernia follows the inguinal canal from the deep to superficial ring and into the scrotum in males (-Fig. 3). It is therefore lateral to Hesselbach's triangle and the inferior epigastric vessels ( - Fig. 4). A femoral hernia is the protrusion of abdominal content through the femoral ring into the femoral canal. It is more commonly seen on the right side and in women. It protrudes inferior to the inguinal ligament/inferior epigastric vessels and is found medial to the femoral vein. Because of its position, it forms lateral to the pubic tubercle and commonly compresses the femoral vein (-Fig. 5). As such, incidence of strangulation is higher in femoral hernias
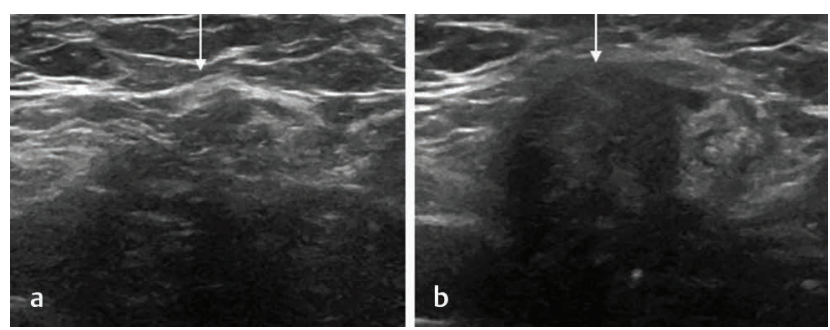

Fig. 2 Direct inguinal hernia. (a) Ultrasound scan (USS) of the inguinal hernia at rest. (b) USS of the inguinal hernia when straining (parasagittal plane). A large protrusion can be seen in keeping with a direct inguinal hernia.
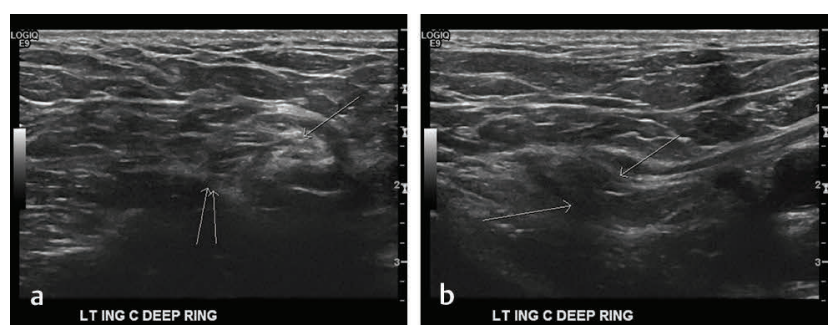

Fig. 3 Indirect inguinal hernia. (a) Double arrows demonstrating deep/internal ring with a single loop of bowel protruding through. (b) Double arrows demonstrating widened borders of the deep inguinal ring, again with a single bowel loop protrusion.
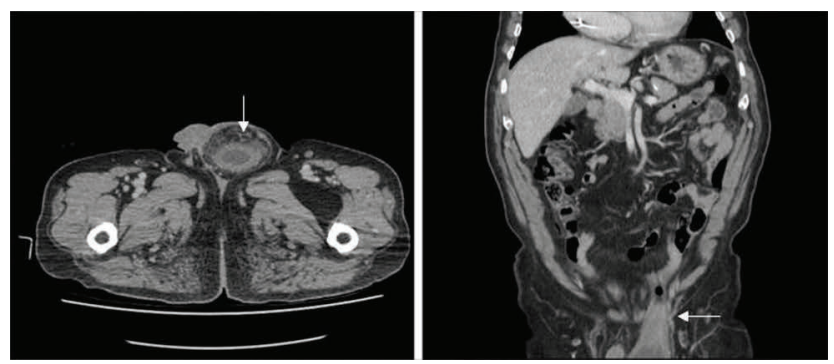

Fig. 4 Inguinal hernia. CT axial and coronal showing left inguinal hernia containing urinary bladder. when compared with other types of groin hernias, so prompt identification and distinction from other hernias are important. The European Hernia Society (EHS) has created a simple classification based on Aachen's classification (-Fig. 6). This can be used as an effective radiologic tool for describing and classifying findings in terms of anatomical locations and size that allows meaningful comparison with the intraoperative findings. This provides good preoperative pretest probability for inguinal and femoral hernias and ensures a common language between radiologists and surgeons. ${ }^{9}$
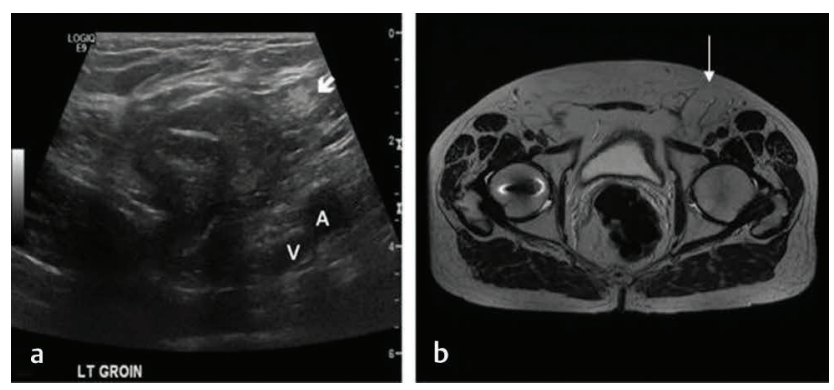

Fig. 5 Femoral hernia. (a) Ultrasound scan (USS) demonstrating femoral hernia containing a loop of ileum. Relationship with the femoral vasculature can be appreciated on this image. (b) T2-weighted MRI of femoral hernia containing momentum only, which was confirmed at surgery.

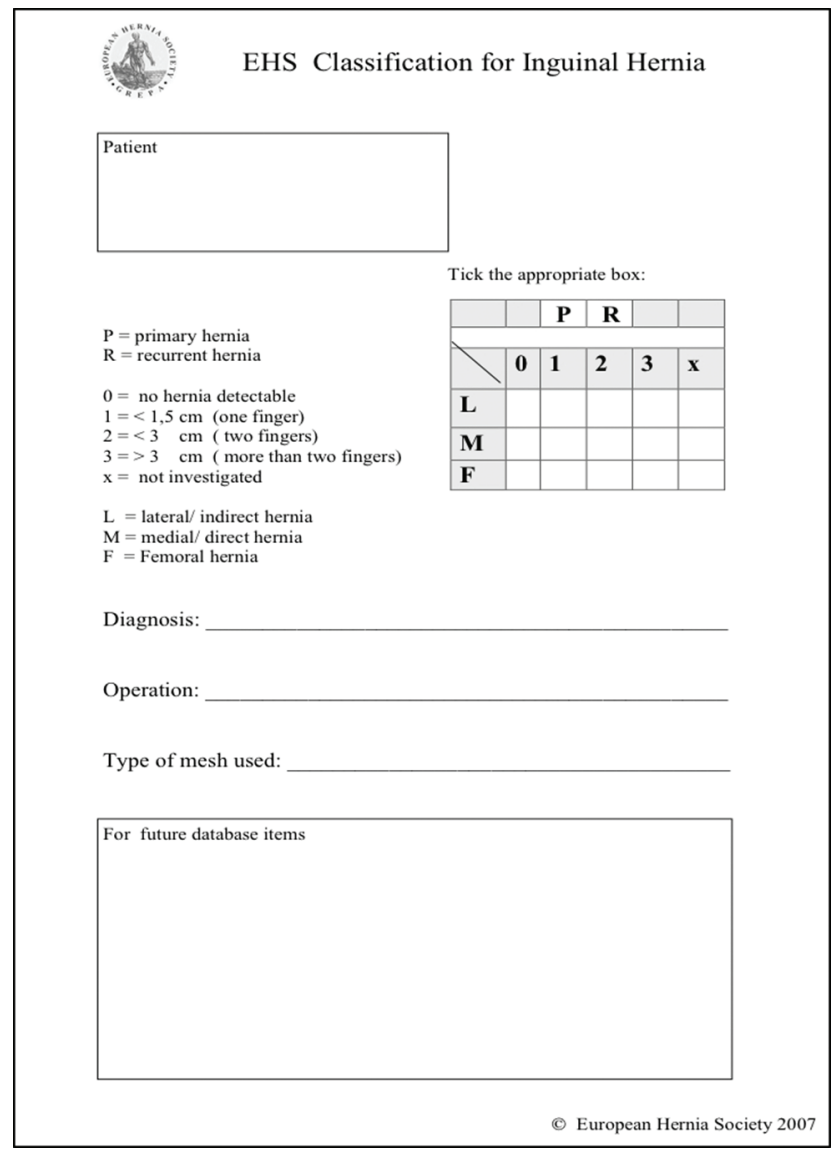

Fig. 6 The European Hernia Society (EHS) classification for inguinal hernia. The EHS created a simple classification based on the Aachen classification. It is use for intraoperative description of the type of hernia to increase the comparison of results in the literature. 


\section{Other Abdominal Wall Hernias}

Ventral hernias are protrusions through the anterior abdominal wall. These can be midline, such as epigastric ( - Fig. 7) and umbilical hernias ( $\mathbf{- \text { Fig. }} \mathbf{8}$ ), or postsurgical such as parastomal and incisional hernias. Incisional hernias are caused by dehiscence of the surgical scar causing protrusion of abdominal content. It is a relatively common hernia, typically occurring after laparotomy (-Figs. 9, 10). A paraumbilical hernia is a midline ventral abdominal wall hernia that occurs near the umbilicus along the linea alba. It is usually related to diastasis of the rectus abdominis muscle. The EHS has also developed a classification system for different parastomal hernias (-Fig. 11). Previous classifications have included clinical, radiologic, and intraoperative findings, with each subgroup having different definitions. As demonstrated by

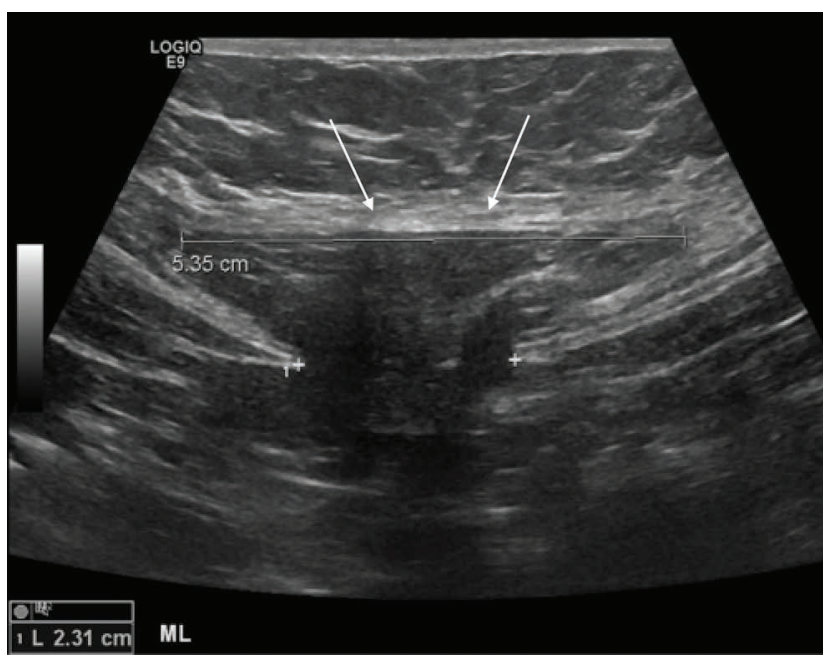

Fig. 7 Epigastric hernia. Ultrasound scan (USS) demonstrating a $2-\mathrm{cm}$ defect in the linea alba with a $5.3-\mathrm{cm}$-wide hernia sac containing omentum.

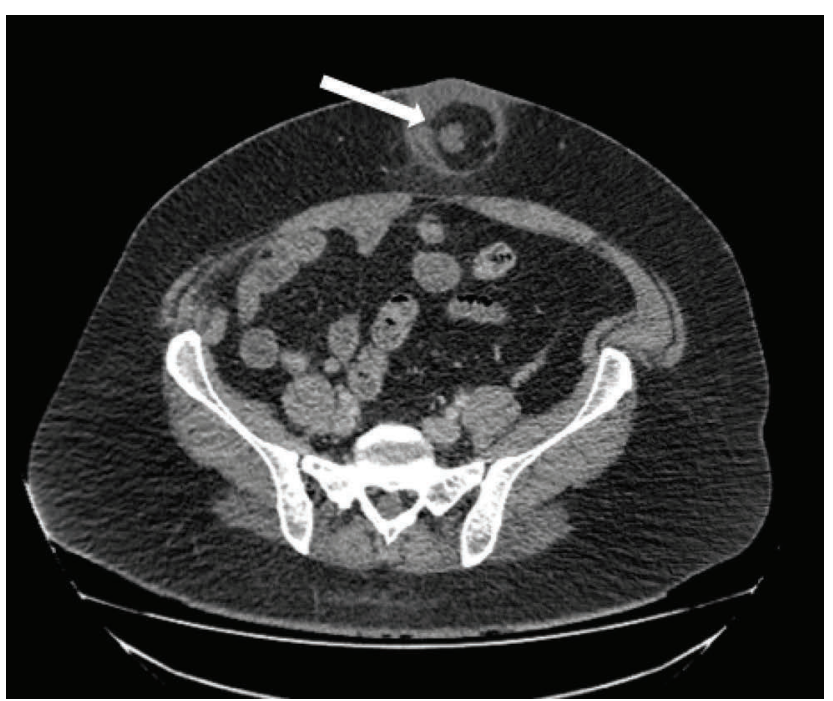

Fig. 8 Umbilical hernia containing peritoneal nodule. Axial contrast-enhanced computed tomography (CT) demonstrating an umbilical hernia in a female patient. Note the nodule within the sac (arrow). The patient was found to have ovarian cancer, and this was the only peritoneal metastasis. the EHS classification of inguinal hernias, this classification system allows different studies to achieve comparable evidence-based therapeutic guidance ${ }^{10}(-$ Fig. 12). The size of the parastomal hernia and presence of a concomitant

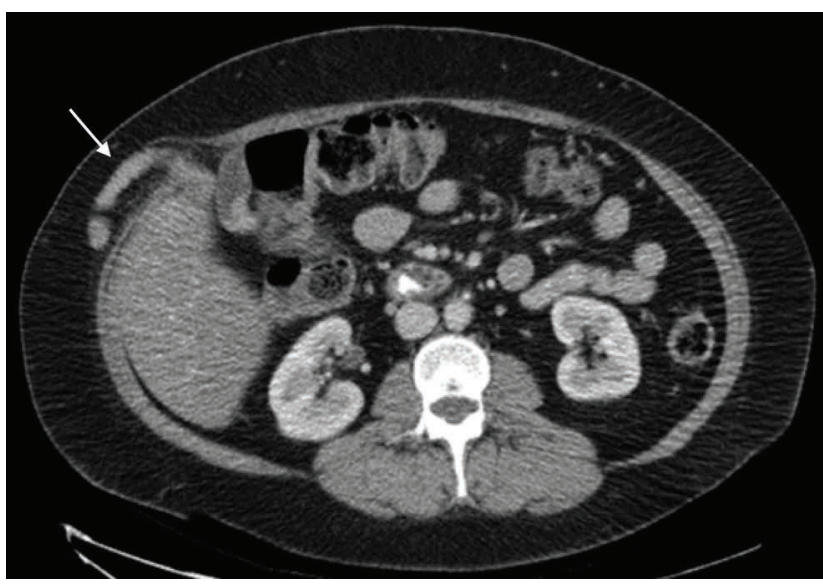

Fig. 9 Port site hernia. Axial computed tomography (CT) showing protrusion of a small bowel loop through previous laparoscopy port site (arrow).

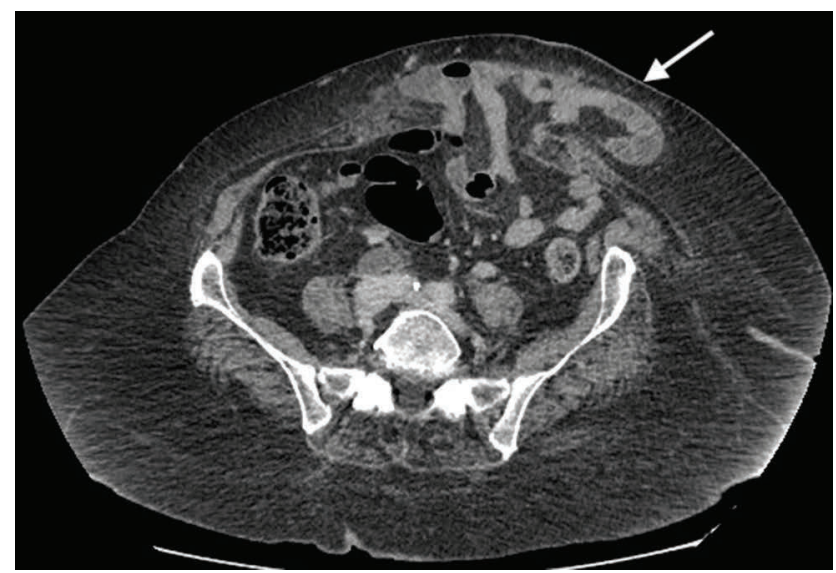

Fig. 10 Incisional hernia. Axial contrast-enhanced computed tomography (CT) demonstrating a large para-midline incisional hernia defect. The sac contains numerous loops of unobstructed small bowel.

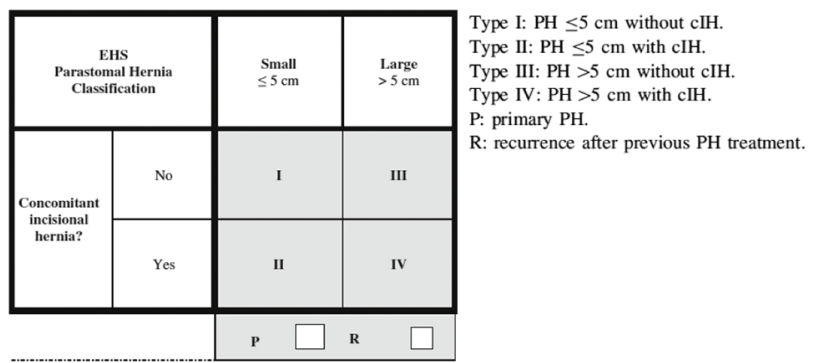

Fig. 11 European Hernia Society (EHS) grid for parastomal hernia $(\mathrm{PH})$ classification. Previously published classification of parastomal hernias were based on clinical, radiologic, and intraoperative findings, with different subgroups having variable definitions. The EHS parastomal hernia classification has been designed to improve the ability to compare different studies and their results. The goal is to provide evidence-based therapeutic guidelines. 
incisional hernia are the most important factors in the surgical management of these patients, the large parastomal hernias with concomitant incisional hernias being the most
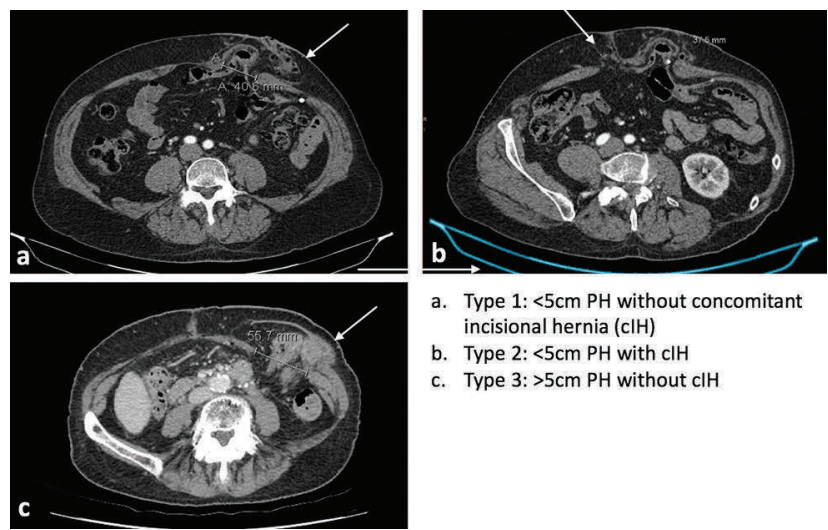

a. Type 1: $<5 \mathrm{~cm}$ PH without concomitant incisional hernia $(\mathrm{clH})$

b. Type $2:<5 \mathrm{~cm}$ PH with $\mathrm{clH}$

c. Type $3:>5 \mathrm{~cm}$ PH without $\mathrm{clH}$

Fig. 12 Parastomal hernia (PH) types according to European Hernia Society (EHS) classification. (a) Type 1: Axial computed tomography (CT) demonstrating $<5 \mathrm{~cm}$ left $\mathrm{PH}$ without concomitant incisional hernia. (b) Type 2: Axial CT demonstrating $<5 \mathrm{~cm}$ PH with a concomitant incisional hernia. (c) Type 3: Axial CT demonstrating $>5 \mathrm{~cm} \mathrm{PH}$ without concomitant incisional hernia.
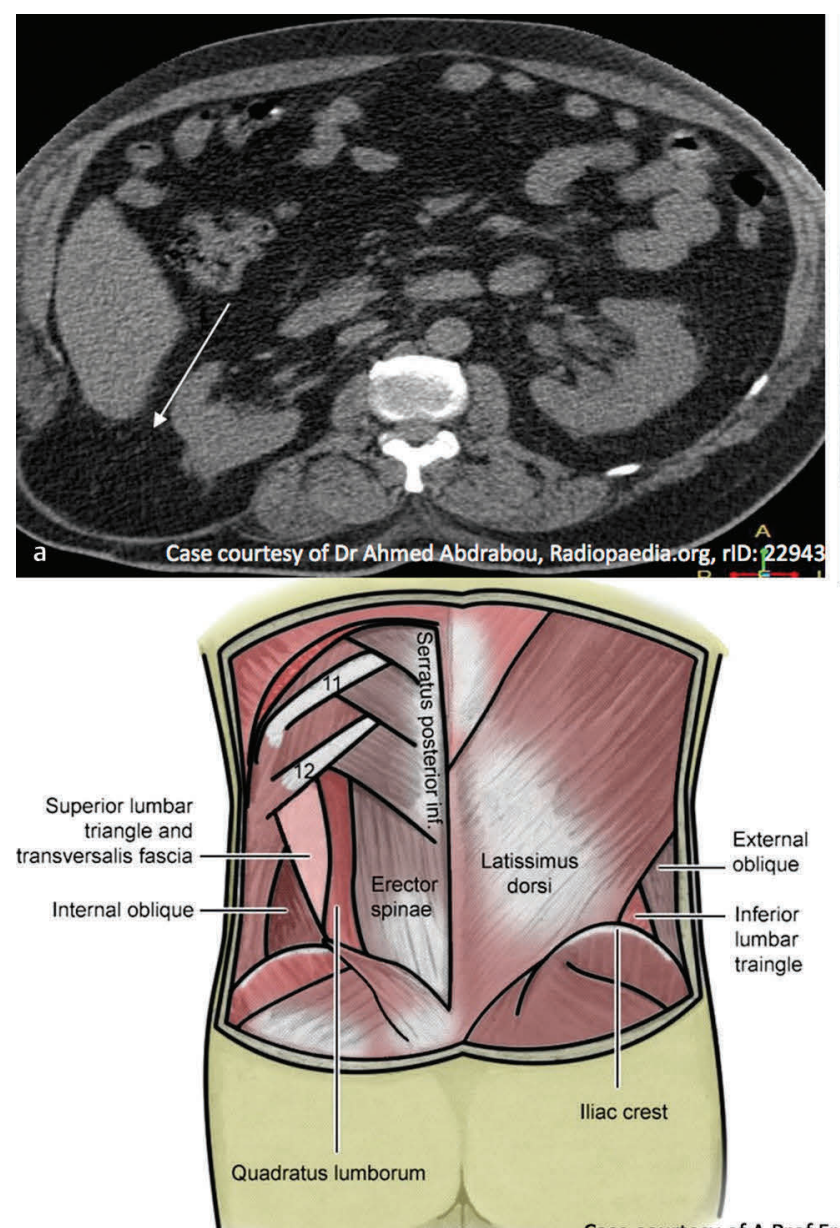

Case courtesy of A.Prof Frank Gaillard, Radiopaedia.org, rID: 12934

c

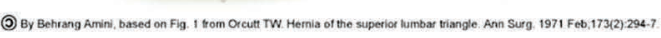

d
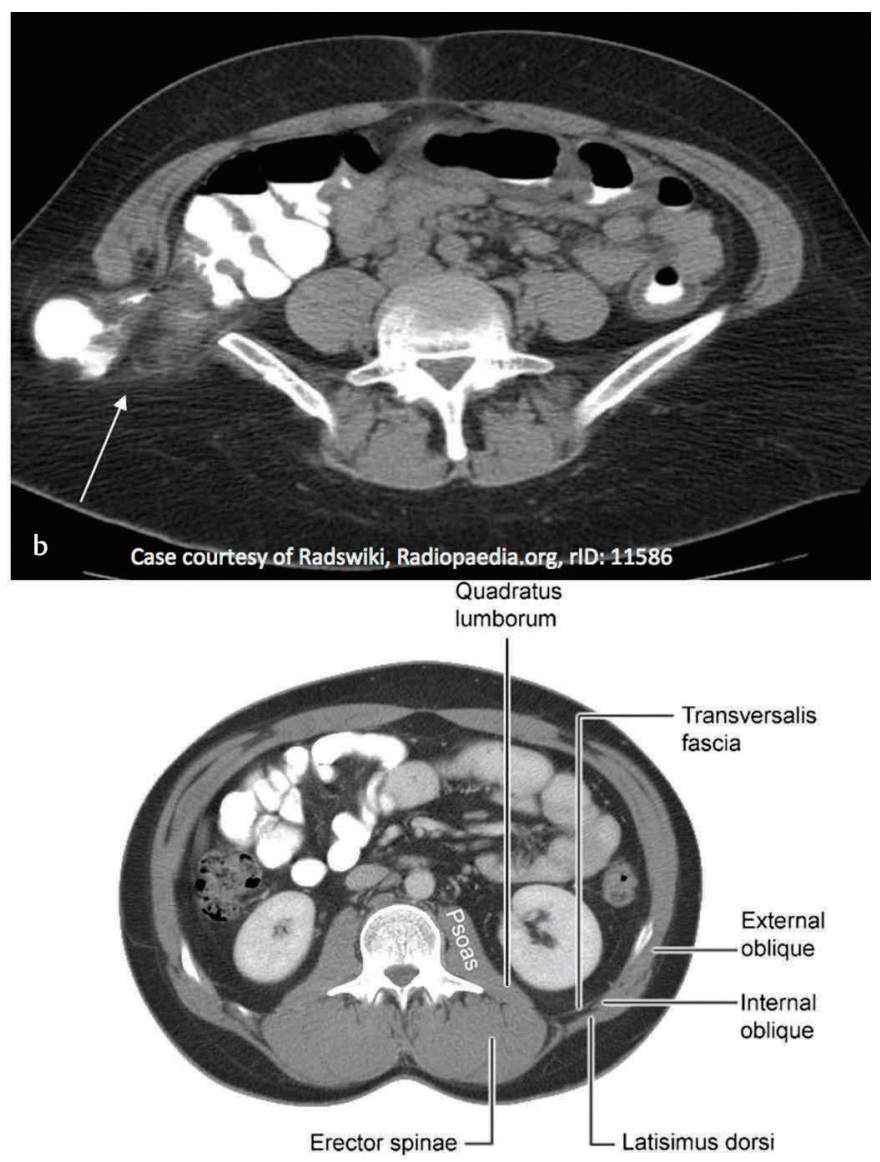

Fig. 13 Lumbar hernia. (a) Axial computed tomography (CT) demonstrating right-sided superior lumbar hernia containing intraabdominal fat only. (b) Axial CT demonstrating right-sided inferior lumbar hernia containing a loop of large bowel. (c) Diagram of lumbar hernia anatomy. (d) Annotated axial CT to show lumbar hernia site anatomy. 


\section{Complications}

Hernias are the second most common cause of small bowel obstruction after adhesions. ${ }^{14}$ Findings include a transition point located at the hernia with proximal small bowel dilatation $^{15}$ (-Fig. 17). Hernia incarceration is predominantly a clinical diagnosis when a hernia is found to be irreducible. It implies the hernia neck is too narrow for the content to return back into the abdominal cavity ${ }^{3}$ (-Fig. 18). Hernia strangulation leads to the compromise of blood supply to the hernia sac contents causing bowel ischemia. This usually occurs when herniated small bowel has an obstructed afferent and efferent limb into the hernia known as a closed-loop obstruction. Imaging features include free fluid, mesenteric

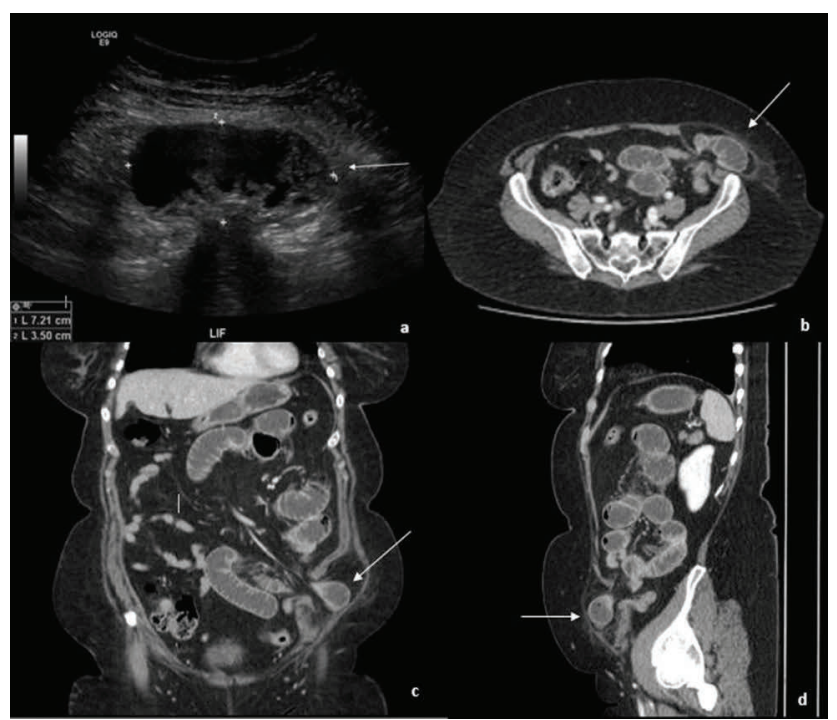

Fig. 14 Spigelian hernia. Patient presented with left flank mass, nausea, vomiting, and abdominal distension. (a) Ultrasound (US) image demonstrating a loop of small bowel in the left abdominal wall subcutaneous tissue. (b) Axial computed tomography (CT) confirms a Spigelian hernia with small bowel obstruction. (c) Reconstructed coronal images demonstrate the Spigelian hernia with small bowel obstruction. (d) Reconstructed sagittal images demonstrating the Spigelian hernia with small bowel obstruction.

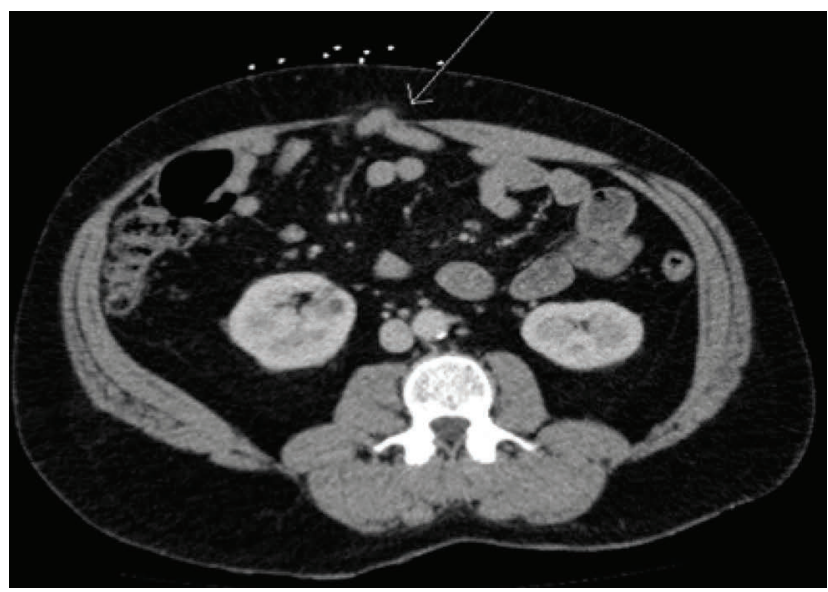

Fig. 15 Richter's hernia. Axial computed tomography (CT) showing antimesenteric bowel wall herniation without compromising the bowel lumen in keeping with Richter's hernia. engorgement, intramural gas, and poor bowel wall enhancement ( Fig. 19). Hyperdense bowel wall can occasionally be seen on unenhanced CT secondary to mural haematoma., ${ }^{3,16}$ Evidence of mesenteric stranding and free fluid may indicate impending strangulation and necessitates early operative intervention to avoid ischemic complications.

The role of the imaging extends also into the postoperative period when complications can and do occur. This,
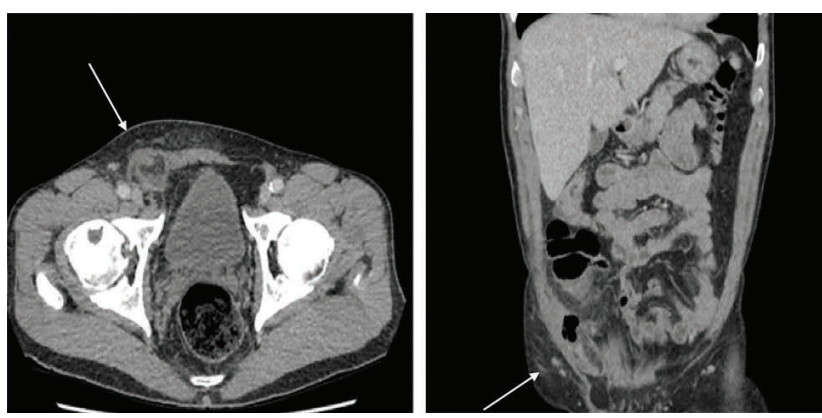

Fig. 16 Amyand's hernia. Axial and coronal computed tomography (CT) showing right-sided inguinal hernia containing inflamed vermiform appendix (acute appendicitis). Findings are consistent with Amyand's hernia.

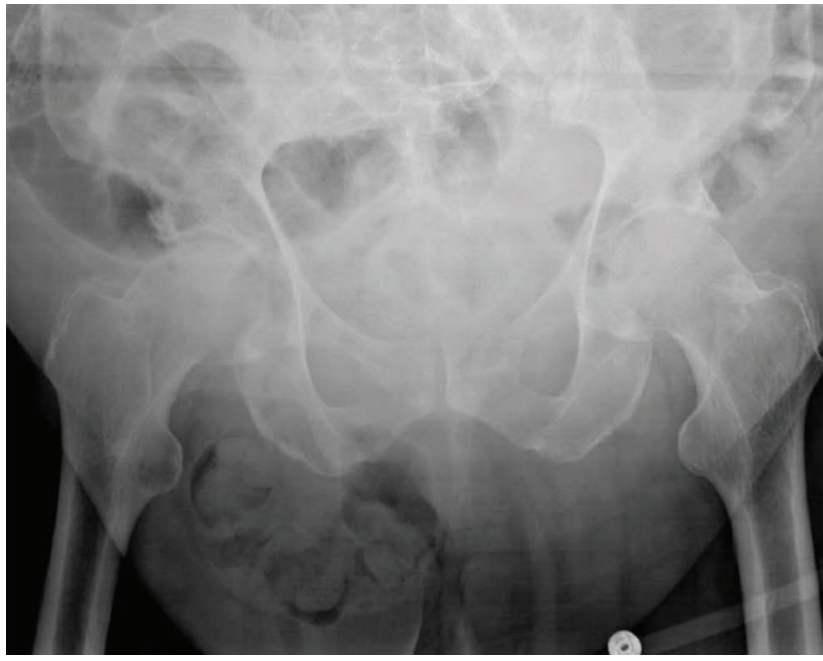

Fig. 17 Obstruction. Abdominal radiograph showing dilated small bowel loops. Note the gas and feces containing small bowel loops below the inguinal ligament in keeping with an obstructed indirect right inguinoscrotal hernia, confirmed at laparotomy.
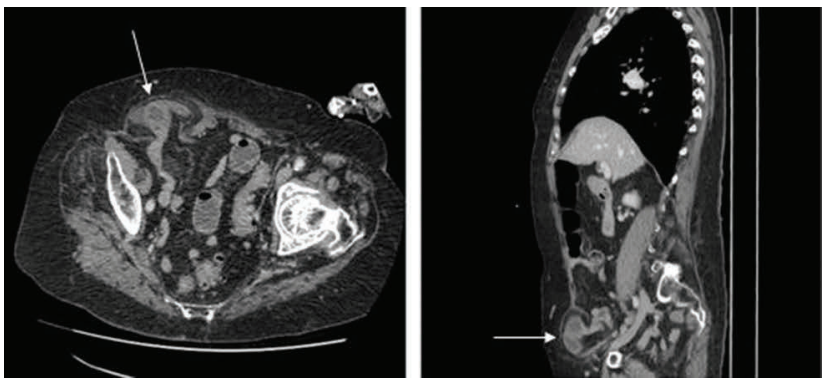

Fig. 18 Incarcerated inguinal hernia. Axial and sagittal CT demonstrating inguinal hernia containing a loop of small bowel. There is mild bowel wall thickening with extraluminal free fluid consistent with incarcerated inguinal hernia. 

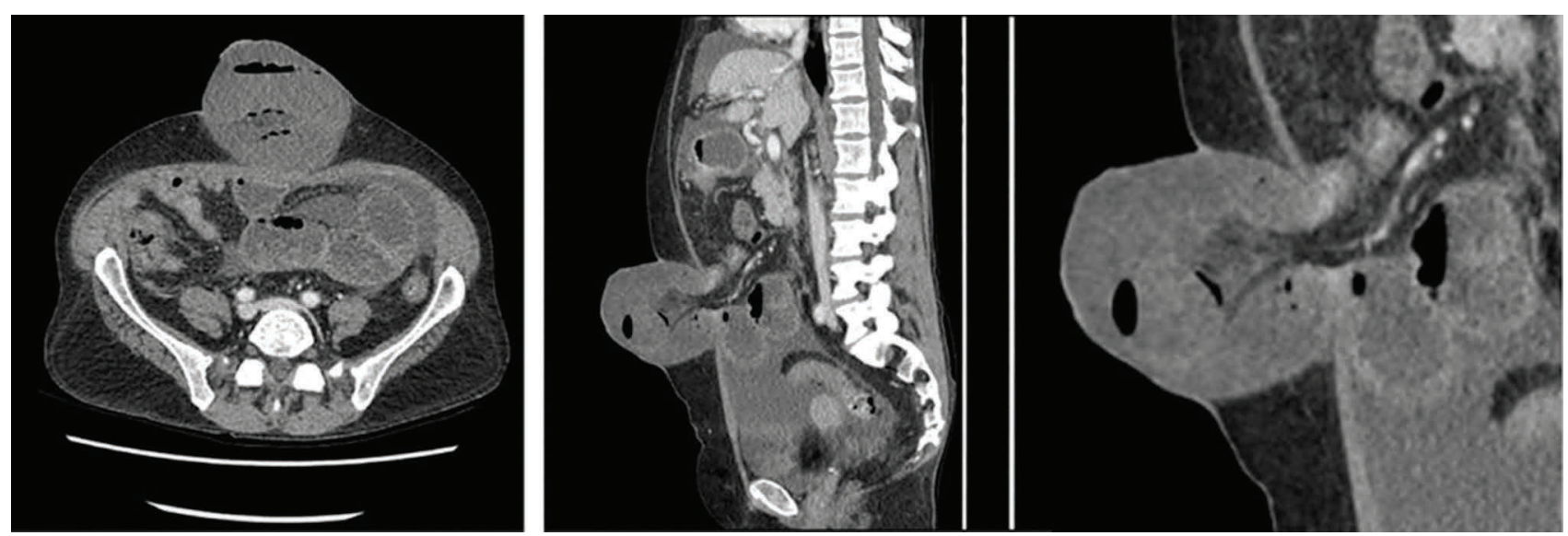

Fig. 19 Strangulated inguinal hernia. A 50-year-old woman who presented with tender umbilical lump. Axial and sagittal computed tomography (CT) images showing an umbilical hernia containing a C-shaped loop of small bowel (closed loop obstruction) with adjacent free fluid, fat stranding, and mesenteric engorgement. There is reduced bowel wall enhancement with intramural gas. Findings are in keeping with strangulated umbilical hernia with gangrenous small bowel.

understandably, can create anxiety among patients and surgeons equally. These complications include recurrent hernias, mesh-associated infections, or bowel mesh adhesions. Some of these may be too complex to diagnose and assess clinically and may need re-intervention. In some cases (if not most) re-look surgery without a certain diagnosis is not advisable. In such cases, imaging can confirm these clinical suspicions and further characterize the findings aiding preoperative planning.

\section{Conclusion}

Abdominal wall and lumbar hernias are common conditions presenting often to general practitioners and surgeons. Presentation is commonly with a lump that may be new or exacerbated by physical activity. Hernias can present acutely due to complications such as bowel obstruction or strangulation. Historically, corrective surgeries were performed based on clinical signs and symptoms; however, with imaging now widely and easily available, more clinicians are opting for diagnostic imaging prior to surgery both for confirmation and planning. It is thus important for radiologists to be able to identify different types of hernias correctly and be able to communicate information that is useful for the clinicians in their management of hernias.

For each hernia, we have identified its typical features, imaging findings in various modalities, and its associated complications to guide radiologists to correctly identify the hernias to expedite its treatment.

\section{Conflict of Interest}

None.

\section{References}

1 Fitzgibbons RJ Jr, Forse RA. Clinical practice. Groin hernias in adults. N Engl J Med 2015;372(8):756-763

2 Primatesta P, Goldacre MJ. Inguinal hernia repair: incidence of elective and emergency surgery, readmission and mortality. Int J Epidemiol 1996;25(4):835-839
3 Aguirre DA, Santosa AC, Casola G, Sirlin CB. Abdominal wall hernias: imaging features, complications, and diagnostic pitfalls at multi-detector row CT. Radiographics 2005;25(6):1501-1520

4 Hair A, Paterson C, Wright D, Baxter JN, O'Dwyer PJ. What effect does the duration of an inguinal hernia have on patient symptoms? J Am Coll Surg 2001;193(2):125-129

5 Poelman MM, van den Heuvel B, Deelder JD, et al. EAES Consensus Development Conference on endoscopic repair of groin hernias. Surg Endosc 2013;27(10):3505-3519

6 Miller J, Cho J, Michael MJ, Saouaf R, Towfigh S. Role of imaging in the diagnosis of occult hernias. JAMA Surg 2014;149(10):1077-1080

7 van den Berg JC, de Valois JC, Go PM, Rosenbusch G. Detection of groin hernia with physical examination, ultrasound, and MRI compared with laparoscopic findings. Invest Radiol 1999;34(12):739-743

8 van den Berg JC, de Valois JC, Go PM, Rosenbusch G. Groin hernia: can dynamic magnetic resonance imaging be of help? Eur Radiol 1998;8(2):270-273

9 Miserez M, Alexandre JH, Campanelli G, et al. The European hernia society groin hernia classification: simple and easy to remember. Hernia 2008;12(3):335

10 Śmietański M, Szczepkowski M, Alexandre JA, et al. European Hernia Society classification of parastomal hernias. Hernia 2014;18(1):1-6

11 Baker ME, Weinerth JL, Andriani RT, Cohan RH, Dunnick NR. Lumbar hernia: diagnosis by CT. AJR Am J Roentgenol 1987; 148(3):565-567

12 Harrison LA, Keesling CA, Martin NL, Lee KR, Wetzel LH. Abdominal wall hernias: review of herniography and correlation with cross-sectional imaging. Radiographics 1995;15(2):315-332

13 Mittal T, Kumar V, Khullar R, et al. Diagnosis and management of Spigelian hernia: a review of literature and our experience. J Minim Access Surg 2008;4(4):95-98

14 Macari M, Megibow A. Imaging of suspected acute small bowel obstruction. Semin Roentgenol 2001;36(2):108-117

15 Boudiaf M, Soyer P, Terem C, Pelage JP, Maissiat E, Rymer R. $\mathrm{Ct}$ evaluation of small bowel obstruction. Radiographics 2001;21(3):613-624

16 Yu CY, Lin CC, Yu JC, Liu CH, Shyu RY, Chen CY. Strangulated transmesosigmoid hernia: CT diagnosis. Abdom Imaging 2004;29(2):158-160 